\title{
Radiological characterisation of nuclear waste-the role of representative sampling
}

\author{
Robin Tuerlinckxa and Kim Harry Esbensen ${ }^{\text {b }}$ \\ aBelgoprocess, Dessel, Belgium \\ ${ }^{b} \mathrm{KHE}$ Consulting, Copenhagen, Denmark
}

During the last year, Belgian nuclear waste processing has been introduced to the practical application of the Theory of Sampling (TOS). This immediately gave rise to important insights leading to significant potential improvements in the field of radioactive waste characterisation. As in many other industry sectors, nuclear waste characterisation is fraught with traditional grab sampling procedures that obviously cause trouble by increasing the total sampling plus analytical uncertainty associated with categorisation of waste materials unnecessarily; this can easily translate into waste mischaracterisation. A pilot project was carried out which challenged today's common practices in nuclear waste characterisation (all internationally accepted as described in Reference 11) with an alternative TOS-compliant methodology. For some commonly encountered waste materials, an experimental setup was developed to gain insights into the typical heterogeneity distribution of key analytical parameters and how to counteract their influence. This has allowed us to design a significantly improved industrial sampling procedure, which is being developed over a very short time span.

\section{DOI: $10.1255 /$ sew.2021.a56}

(c) 2021 The Authors

Published under a Creative Commons BY-NC-ND licence

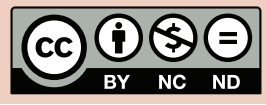

The new material-dependent, threepart process (visual inspection, sorting, representative sampling for analysis) is described and illustrated together with several improvement potentials.

\section{Background}

Belgoprocess is the industrial operator of the waste management facilities owned by the National Agency for Radioactive Waste and Enriched Fissile Materials (ONDRAF/NIRAS) in Belgium, responsible for nuclear waste management and site remediation at two historical sites in Belgium. Belgoprocess was founded after the European pilot reprocessing plant EUROCHEMIC terminated its activities in the early 1980s. The company is also in charge of the decommissioning and remediation of obsolete nuclear facilities on its sites, as well as the characterisation of legacy wastes in compliance with all relevant safety requirements.

Preparing legacy waste for end-stage storage is a very complex task, necessitating a proven ability to conduct reliable categorisation of preliminary storage units (drums, containers, stocks ...) commensurate with official waste acceptance criteria (WAC), as well as paying particular attention to long-term safety criteria. However, some of the most critical radionuclides, as far as long-term safety is concerned, are difficult-to-measure (DTM), meaning that the application of "remote" non-destructive assay (NDA) is insufficient to cover all aspects associated with these tasks. A commonly used approach is, therefore, a combination of NDA and the use of scaling factors, which are based on empirical correlations between DTM radionuclides and other, easy-to-measure (ETM) key radionuclides-whilst assuming a degree of homogeneity of activity distribution of numerous radionuclides over the investigated lot.

A new fit-for-purpose characterisation process is presently being developed at Belgoprocess to cope with the very low activity concentrations that are specified in the WAC. For this project, waste characterisation protocols in Belgium were introduced to the TOS and a robust, representative sampling process has been developed over the last year. Several Replication Experiments (REs) were conducted to survey and quantify the intrinsic heterogeneity of selected typical materials for a range of critical WAC parameters. There are many specific analytical requirements that must be considered when applying standard TOS principles. Despite all first results indicating that radiological contamination is indeed very heterogeneously distributed, a dedicated primary sampling process has been designed and implemented. This shows promising results as testified by significantly better accuracy than the commonly used NDA techniques.

\section{Nuclear waste management challenges (not your ordinary heterogeneous lots]}

Historical records contain a lot of relevant information, but unfortunately of different systematic content and quite often insufficient to demonstrate compliance 
with the very low concentration limits stipulated in today's WAC for near-surface disposal. It is important to recognise that the WAC have evolved markedly in the last decades, for example indicating that sorting efforts with respect to different physico-chemical compositions and contents have not been performed to the desired level over the same period. The present approach will not result in sufficient information regarding longterm safety aspects, which is critical for the near-surface disposal site. As an example, the limit on cellulose content has been reduced significantly over the last 20 years, today reaching a level of a maximum of $100 \mathrm{~g}$ in the finalised WAC to be complied with today. For historical waste, this inevitably gives rise to the necessity of a thorough visual inspection of its content. All this adds impetus to the development of a new, more comprehensive approach.

In the Belgian waste disposal concept's safety case, the presence of cellulose is of equal importance as the radioactivity content. This is due to the potential complexing properties of cellulose's degradation products (e.g. isosaccharinic acid) in high alkaline environments, which could increase the mobility of radioactivity in the disposal site.

Worldwide, there is consensus that mobile DTM radionuclides like ${ }^{129} \mathrm{I}$ and ${ }^{99} \mathrm{Tc}$ are key for long-term safety. The relevance of these DTMs is stipulated by the low activity concentrations that are allowed in WAC related to a variety of disposal frameworks.

Another concern is that the current description of radiological content is incomplete or the existing data are, in some cases, unsatisfactory with respect to today's WAC. Our first survey drums were characterised for radiological content using the standards for waste characterisation that were valid at the time of production, i.e. decidedly not at the levels expected today. A primary challenge lies in obtaining a sufficiently reliable characterisation, taking account of the radionuclides which are of great concern for long-term safety, e.g. ${ }^{241} \mathrm{Am}$, ${ }^{129} \mathrm{I},{ }^{90} \mathrm{Sr}$ and plutonium isotopes, based on a theoretical safety case.

Best practices for radioactive waste characterisation are extensively described in the literature. However, it is rarely the case that these can be matched to the desired accuracy associated with the current WAC that must be followed, especially for historical waste where there is little information about the specific origin of the contamination encountered. In general, radioactive waste characterisation of legacy waste is associated with unavoidable, significantly large uncertainties-up to several orders of magnitude, due to severe-to-extreme heterogeneity "mixed-waste" effects and specific measurement restrictions (e.g. complicated analytical background effects). The present concentration limits for final near-surface disposal raises significant challenges in both material characterisation and analysis.

The majority of the parameters of interest are impossible to measure accurately in a non-destructive way, e.g. drum-level measurement using "remote" NDA gamma spectrometry (some illustrations of commercially available equipment are shown in Figure 1). So, interest has grown towards the field of process sampling which is frequently applied in a wide range of different technological and industrial contexts. This column describes the reasons behind, and basis for, development and application of process sampling approaches guided by the TOS framework for the purpose of radioactive waste characterisation under stringent boundary conditions. This was the reason for initiating collaboration with KHE Consulting. In the present pilot project, the aim is to establish practical ways to implement the TOS' principles for sampling of radioactive waste for today's highly conscribed characterisation purposes. The aim is to design, construct, implement and to start using fit-for-purpose representative sampling processes only.

Below is described a challenging baseline experiment in which the intrinsic waste heterogeneities are surveyed and quantified for the first time. This has led to development of a practical approach based on calibrated composite sampling procedures and commensurate equipment (Figure 2).

\section{The Theory of Sampling}

Representative sampling is a common prerequisite for many analytical endeavours, including tasks associated with waste characterisation-not only for nuclear waste. Despite the obvious advantage of only analysing representative samples, in the literature, as well as in practice, there sadly are lots of examples and cases where this demand is tacitly, unwittingly or wilfully neglected. Especially in the case of significantly heterogeneous materials, which legacy nuclear waste materials undoubtedly are, one must come to grips with the fact that the impact of the primary sampling process to the overall accuracy is at least of an order-of-magnitude larger than the analytical uncertainty (and can under conditions of severe heterogeneity be much larger still). The approach in the present nuclear waste management project, therefore, takes its point of departure on the basis of the TOS as

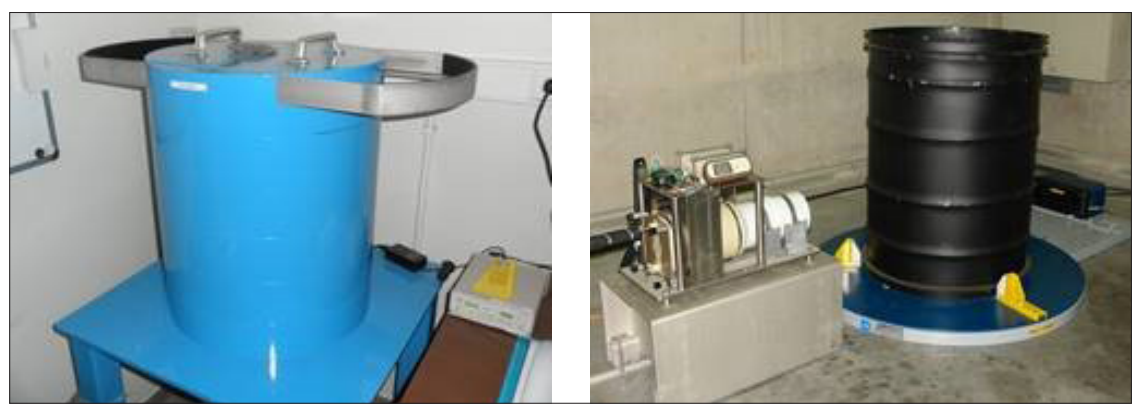

Figure 1. Typical gamma spectrometry instrumentation used in radioactive waste characterisation: lab-scale geometry (left) versus drum-level measurement (right). 


\section{SHAMPLINE GOLEN}

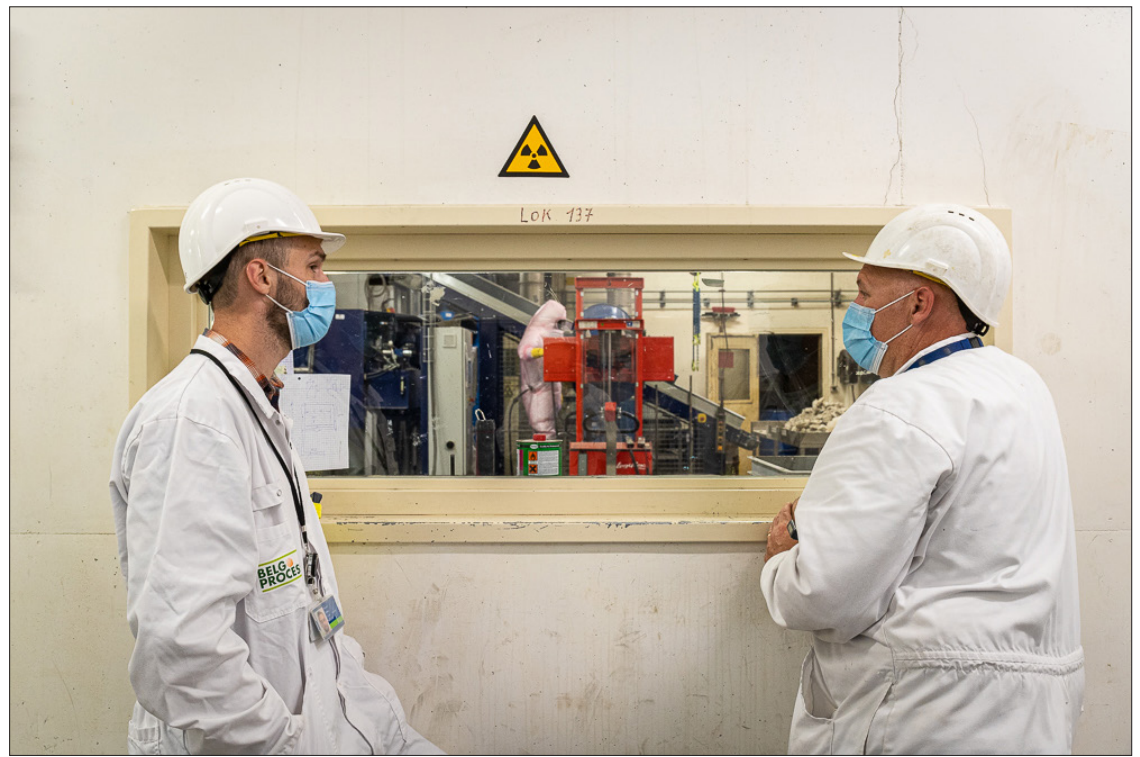

Figure 2. A look into a hands-on hot cell, where manual interventions and handling of radioactive contaminated materials can be carried out in safe working conditions

the necessary-and-sufficient guide for design, development and implementation of a reliable, representative sampling methodology.

The TOS is a universal, scale-invariant framework for understanding sampling and all potential associated errors. One of the TOS' primary principles states that representative sampling of a heterogeneous material (lot) must be in the form of a composite sampling process, involving taking a sufficient number of increments $(Q)$ in such a way that the entire lot volume has even odds for being sampled. It is quite out of the question to use the single-increment extrac tion sampling procedure known as grab sampling. The entire 70 years of experience of the TOS has presented an overwhelming array of evidence for the unavoidable, non-representative character of grab sampling of heterogeneous materials and lots.

Another cardinal rule of the TOS is that all sampling procedures "from-lotto-aliquot" are multi-stage procedures, necessary to counteract the various effects stemming from the many manifestations of lot heterogeneity at several scales-there is both compositional heterogeneity, spatial heterogeneity as well as grain-size distribution heterogeneity to be considered. In complicated situations, up to seven types of sampling error can arise as a specific sampling process interacts with a heterogeneous material, but most ordinary situations are simpler. Two sampling errors arise because of the heterogeneity of the sampling target and four so-called bias-generating errors can be produced by the sampling process itself-if the potential effects of these errors are not properly understood, reduced and/or eliminated. In recent years, the TOS has been presented and explained at all levels of interest, from initial introductions to complete textbooks. ${ }^{1-7}$

The replication experiment When complying with all the basic TOS principles, there is only one free parameter for composite sampling: the number of increments $(Q)$ needed to manage and control the resulting total sampling error. Lot heterogeneity characterisation (LHC) typically consists of a practical experiment with the purpose of determining the $Q$ needed to be able to document a fit-for-purpose representative sampling process. At the start of the present project, a series of REs were deployed as empirical investigations of the effective lot/material heterogeneities affecting primary sampling procedures. These baseline experiments were performed on typical radioactive waste materials, which have been selected and sorted out during physical inspection and repackaging of a limited number of legacy 200-L drums to develop a first idea of the level of heterogeneity of the radiological contamination encountered. These experiments were performed using existing basic equipment, without performing any major process adaptions in advance.

For each material/drum selected, the waste-equivalent to the volumetric content of a 200-L drum-was first reduced in particle size by effective shredding (if/where needed), then transformed to a moving 1-D conveyor belt in a hot-cell environment and eventually sorted and sampled by three alternative procedures, each procedure performed in ten-fold repetition. All samples taken in the REs were analysed by lab-scale gamma spectrometry. Using the same analytical procedure ensured that the best discrimination between the alternative sampling procedures could be obtained. Gamma spectrometry can be viewed as a standard analysis technique-also a popular screening approach-because of the limited sample preparation necessary.

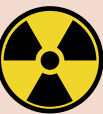
Gamma spectrometry is essentially a non-destructive method allowing screening of gamma radioactivity content. Passive spectrometry of the investigated sample, or drum, allows gamma-emitting radionuclides to be distinguished based on the energy of the emitted photons due to radioactive decay. The most commonly used detector for this type of measurement, both at lab-scale and for whole drums, is a high purity germanium detector. The measurement geometry ("sample preparation") is in all cases identical, a 250-mL sample container filled to the maximum level with suitably mass-reduced target material.

Non-sampled materials are collected in new 200-L drums which are measured directly by drum-level gamma spectrometry. Despite this being the common application in nuclear waste characterisation-often in combination with a nuclide vector approach-this 


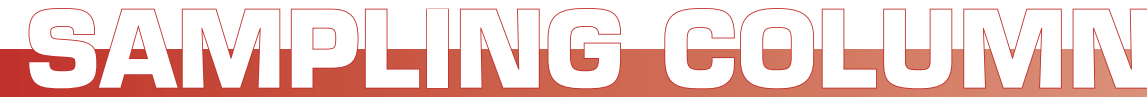
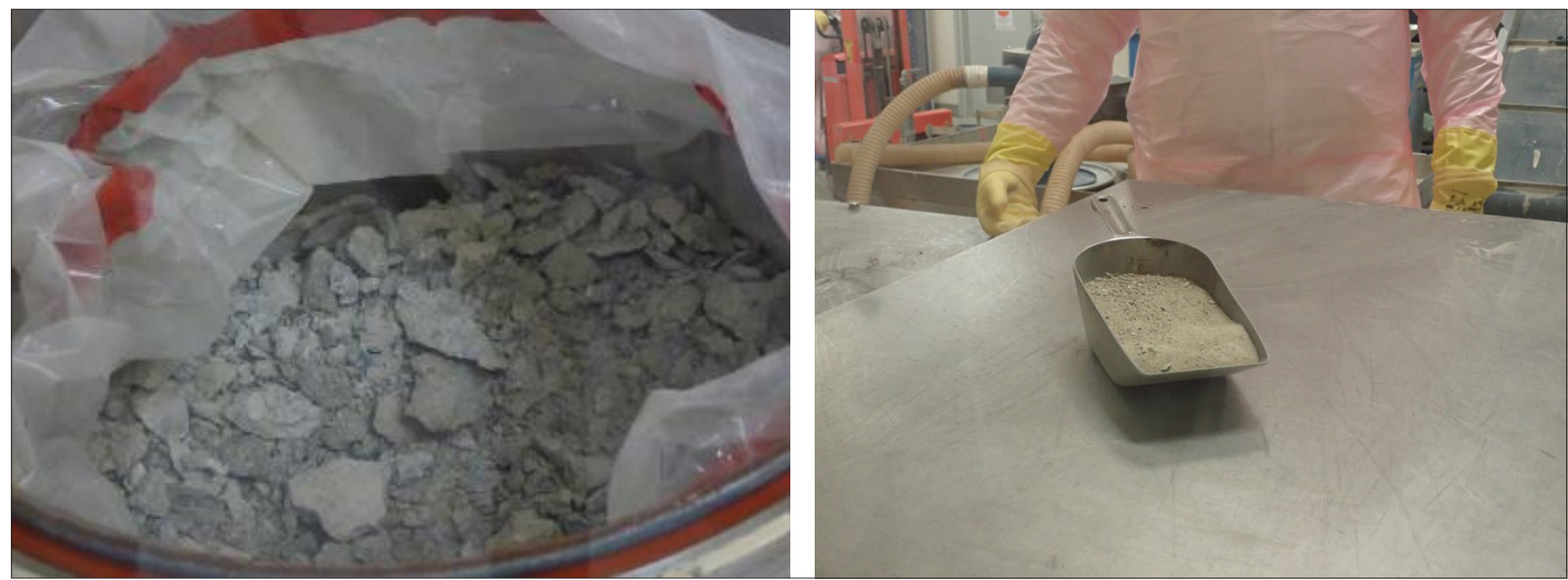

Figure 3. Visual manifestations and typical particle sizes of commonly met with concrete-based materials in nuclear waste. For the radionuclides present amongst other analytes, it is particularly important to understand that material heterogeneity will only seldom give rise to visual manifestations and effects (radioactivity is invisible).

non-destructive measurement technique is nevertheless often associated with significant uncertainties due to the assumption of a homogeneous activity distribution and correlation. For general introductions to radioactivity analysis and related analytes, see References 8 and 9.

\section{Results and process development}

The initial project stages focused on results obtained for selected legacy waste drums with concrete materials. Figure 3 provides an impression of the typical bulk contents which is anticipated in the full project. Drums can contain a broad range of concretelike materials resulting from operations in decommissioning and decontamination.

In the baseline REs in the first stage pilot investigations, TOS-compliant primary sampling was simulated without loss of generality by a 3-D lot transformation of storage drums made into a moving 1-D material stream (loaded onto a conveyor belt). The first stage results show that the typical drum contents can be characterised to the desired level of precision with a 36-increment primary composite

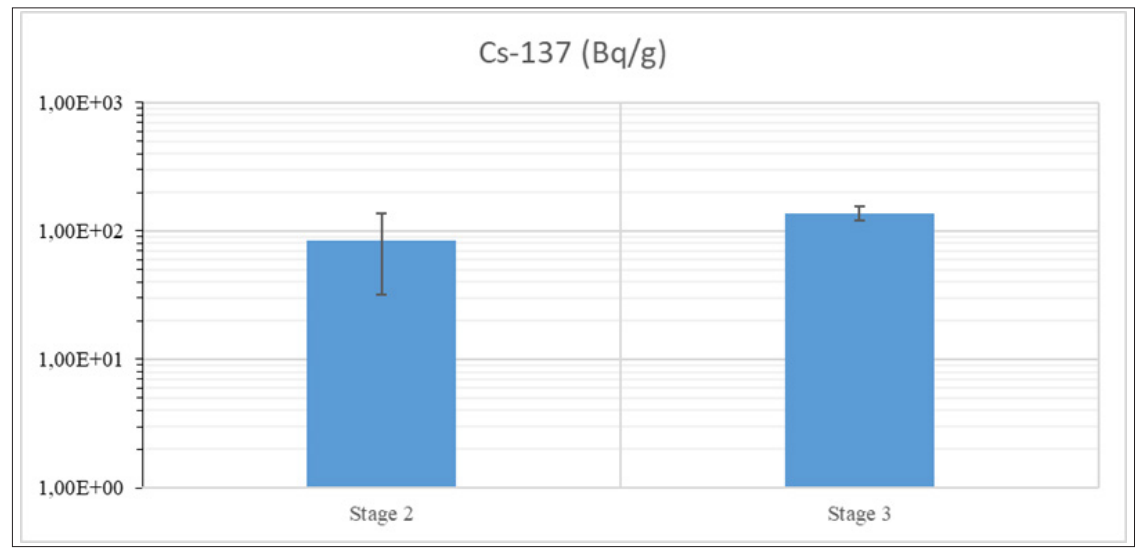

Figure 4. Example of comparison of composite sampling methodologies for representative analysis of ${ }^{137} \mathrm{C}$ in concrete fines (stage 2 is $Q=6$ composite samples; stage 3 is $Q=36$ composite samples). Bars represent the average activity concentration and error bars represent RSV levels. ${ }^{1,2,4}$ sample approach. Whereas grab and composite sampling with $Q=6$ increments distinctly under-performed as evidenced by RSV\% larger than $15 \%,{ }^{1,2,4}$ which had been decided upon as the pilot project threshold level. The full pilot project results, which include comprehensive analytical technique evaluations, are reported elsewhere. ${ }^{10}$

The pilot project manual 1-D process approach will not be feasible at the much larger sample throughputs needed for the full legacy project, however, and especially not with respect to the necessary good practices in radiation protection and cost efficiency. Therefore, a more industrialised process approach is now under development, including the use of a rotary sample divider to do the same composite sampling job as the manual sampling along the linearised 1-D layout.

This type of primary sampler was selected first-and-foremost because it can easily be fitted into a dedicated process layout due to its compact size. But of equal interest, this type of equipment allows the use of a flexible $Q$, for example a very high number of increments for very highly heterogeneous waste types with negligible extraction errors for dry granular materials. The laboratory rotary sample divider is an easy fit-in at the pilot scale sorting and sampling 


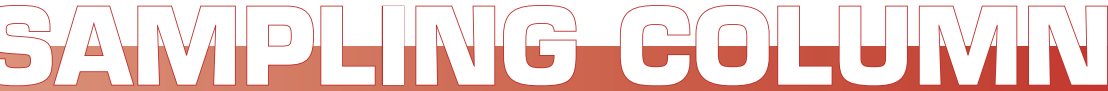

installation, which was built in an existing $\left(\sim 100 \mathrm{~m}^{2}\right)$ intervention cell equipped with a waste shredder for particle size reduction, as seen in Figure 4, and readily available industrial versions (RTD samplers) have shown great stability and durability with respect to the abrasive concrete materials to be processed in high volumes.

\section{Conclusions and prospects}

Earlier, radiological characterisation focused mainly on the unitary approach of measuring the activity level from beta-gamma emitters and total alpha content. With the introduction of isotope-specific concentration limits in the contemporary WAC for both near-surface disposal and (conditional) release, the traditional characterisation methodologies are challenged. We strongly believe that a characterisation process based on material-dependent visual inspection, sorting and representative sampling for analysis will become a valuable tool in future management of a variety of radioactive waste streams. There are many advantages for an updated, WAC-compliant process, but only if based on strict TOS principles and carefully calibrated with respect to specific waste types wherever needed.

Thus, as an addition to traditional non-destructive characterisation, Belgoprocess and KHEC have developed the present Mark 1 pilot installation to deal with the required intensive sorting and representative sampling for contemporary WAC-compliant waste characterisation. Based on the present results from focused extraction of representative samples, the classic drawbacks of commonly used drum-level NDA gamma spectrometry only can now be significantly improved. This will be a great asset for high throughput legacy waste characterisation, or in the case of very heterogeneously distributed analytical parameters in other nuclear waste media, e.g. contaminated soils and sand, which also need to be analysed for very low activity levels.

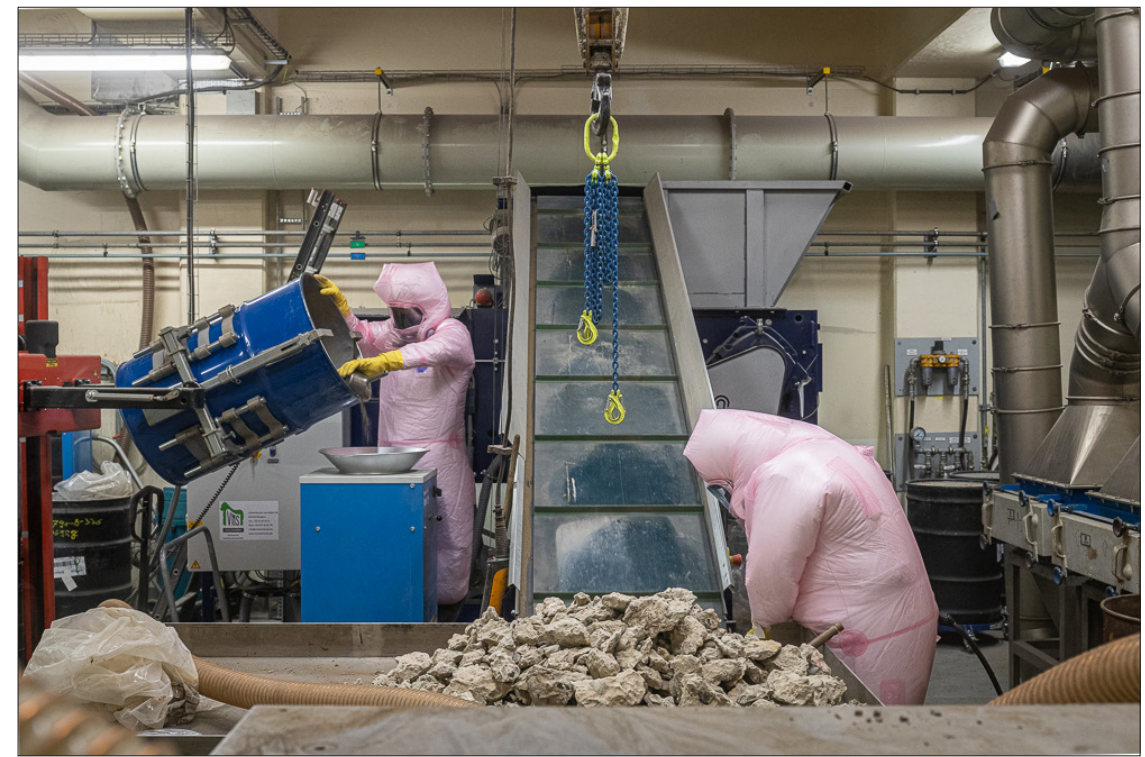

Figure 5. Pilot installation at Belgoprocess dealing with characterisation of concrete containing radioactive waste materials-sorting table and conveyor to shredder (front) and rotary sampler addressing more granulated drum content (left). This pilot experiment equipment setup can easily be scaled up by incorporating industrialised versions of, for example, rotary sample dividers.

This new methodology is complementary to existing, somehow limited characterisation practices because the representative sample is appropriate for direct analysis of DTM radionuclides as well. Besides for radiological characterisation, bona fide representative samples from the nuclear waste realm can also be analysed for chemical characteristics, which are equally critical for certain other nuclear waste streams.

In the next few years, this technique will be applied generally for nuclear waste characterisation aimed at further improvements, for example, regarding upscaling and automation to reach higher processing rates for existing, and possibly new near-future, legacy nuclear waste volumes.

\section{References}

1. K.H. Esbensen, "Materials properties: heterogeneity and appropriate sampling modes", J. AOAC Int. 98(2), 269-274 (2015). https://doi. org/10.5740/jaoacint.14-234

2. K.H. Esbensen, Introduction to the Theory and Practice of Sampling. IM Publications Open (2020). https:// doi.org/10.1255/978-1-906715-29-8

3. F.F. Pitard, Theory of Sampling and Sampling Practice, $3^{\text {rd }}$ Edn.
CRC Press (2019). https://doi. org/10.1201/9781351105934

4. DS3077, Representative SamplingHorizontal Standard. Danish Standards (2013). http://www.ds.dk

5. K. Esbensen and P. Paasch Mortensen, "Process sampling (Theory of Sampling, TOS) - the missing link in Process Analytical Technology (PAT)", in Process Analytical Technology, Ed by K. Bakeev. Wiley-Blackwell, pp. 37-80 (2010). https://doi. org/10.1002/9780470689592.ch3

6. K.H. Esbensen and C. Wagner, "Theory of sampling (TOS) versus measurement uncertainty (MU): A call for integration", Trends Anal. Chem. 57, 93-106 (2014). https:// doi.org/10.1016/j.trac.2014.02.007

7. P. Minkkinen, "Sampling vs analytical error: where the money is ...", Spectrosc. Europe 33(5), 46-50 (2021). https://doi.org/10.1255/ sew.2021.a25

8. G. Gilmore, Practical GammaRay Spectrometry. John Wiley \& Sons (2008). https://doi. org/10.1002/9780470861981

9. G.F. Knoll, Radiation Detection and Measurement. John Wiley \& Sons (2010). https://bit.ly/30sOjuG 
10. R. Tuerlinckx, K. Esbensen and T. Huys, Representative Sampling and Radiological Characterization of Heterogeneous Legacy Waste 22031. WM2022 Conference, 6-10 March 2022, Phoenix, Arizona, USA.

11. ISO 21238:2007, "Nuclear energy - Nuclear fuel technology - Scaling factor method to determine the radioactivity of low- and intermediatelevel radioactive waste packages generated at nuclear power plants", $1^{\text {st }}$ Edn. International Standard Organization (2007). https://www. iso.org/standard/40081.html

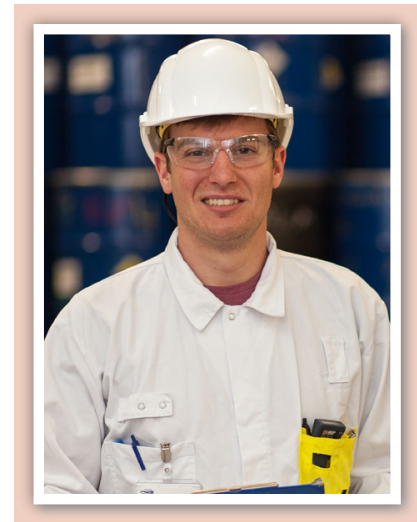

Robin Tuerlinckx graduated as a chemical engineer in 2010 at Hasselt University in Belgium with a specialisation in environmental chemistry and radiochemistry. He started his career in the field of environmental measurements in an analytical lab supporting air quality monitoring and soil remediation. In 2012, Robin joined Belgoprocess in the radioactive waste characterisation team. During the last 10 years he gained experience in a variety of aspects including waste management and non-destructive assay. In 2019 he was triggered by the Theory of Sampling, giving rise to the pleasant introduction to Prof. Esbensen not much later.

(iD_https://orcid.org/0000-0002-0659-7325 robin.tuerlinckx@belgoprocess.be

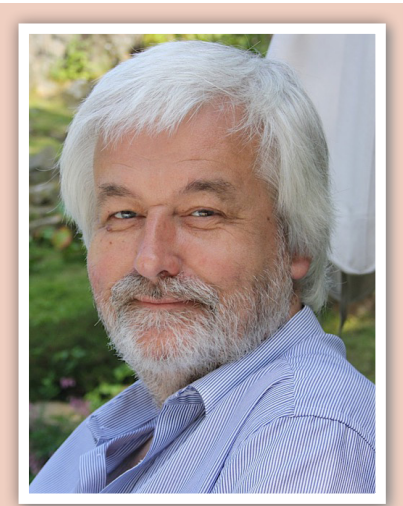

Kim H. Esbensen, PhD, Dr (hon), has been research professor in Geoscience Data Analysis and Sampling at GEUS, the National Geological Surveys of Denmark and Greenland (2010-2015), chemometrics \& sampling professor at Aalborg University, Denmark (2001-2015), professor (Process Analytical Technologies) at Telemark Institute of Technology, Norway (1990-2000 and 2010-2015) and professeur associé, Université du Québec à Chicoutimi (2013-2016). From 2015 he phased out a more than 30year academic career for a new quest as an independent researcher and consultant. But as he could not terminate his love for teaching, he is still very active as an international visiting, guest and affiliate professor. A geologist/geochemist/metallurgist/data analyst of training, he has been working $20+$ years in the forefront of chemometrics, but since 2000 has devoted most of his scientific R\&D to the theme of representative sampling of heterogeneous materials, processes and systems: Theory of Sampling (TOS), PAT (Process Analytical Technology) and chemometrics. He is a member of several scientific societies and has published over 250 peer-reviewed papers and is the author of a widely used textbook in Multivariate Data Analysis (35,000 copies), which was published in its $6^{\text {th }}$ edition in 2018 . He was chairman of the taskforce behind the world's first horizontal (matrix-independent) sampling standard DS 3077 (2013). He is editor of the science magazine TOS forum and this Sampling Column. In 2020 he published the textbook: Introduction to the Theory and Practice of Sampling (impopen.com/sampling).

(iD https://orcid.org/0000-0001-6622-5024

khe.consult@gmail.com 